\title{
BET Bromodomain Proteins as Cancer Therapeutic Targets
}

\author{
Shaokun Shu and Kornelia Polyak \\ Department of Medical Oncology, Dana-Farber Cancer Institute; Department of Medicine, Brigham and \\ Women's Hospital; and Department of Medicine, Harvard Medical School, Boston, Massachusetts 02215 \\ Correspondence: Kornelia_polyak@dfci.harvard.edu
}

\begin{abstract}
Epigenetic regulators are emerging therapeutic targets in a wide variety of human cancers. BET bromodomain proteins have been identified as key regulators of oncogenic transcription factors including MYC; therefore, their inhibition might provide a way to block these "undruggable" targets. Several BET bromodomain inhibitors are in clinical development with promising preliminary findings. However, tumors acquire resistance to these agents in several different ways. In this review, we summarize the role that BET bromodomain proteins play in tumorigenesis as well as the molecular mechanisms underlying therapeutic responses and resistance to their inhibition with emphasis on BRD4 and breast cancer.
\end{abstract}

Epigenetic regulation of gene expression is a dynamic and reversible process that establishes normal cellular phenotypes but also contributes to human diseases including cancer. Epigenetic changes that occur during tumor progression are increasingly recognized as potential therapeutic targets in various human cancer types. Histone modifications (e.g., methylation and acetylation) regulate chromatin structure and transcription, and abnormalities in this process lead to perturbed development and differentiation. The demonstration of frequent somatic mutations in histones and chromatin-modifying factors in a wide range of human tumor types accentuates the importance of epigenetic regulation in tumorigenesis (van Haaften et al. 2009; Dalgliesh et al. 2010; Morin et al. 2011). These regulators are broadly categorized into (1) writersenzymes that catalyze histone or DNA modifications such as histone and DNA methyl transferases, (2) erasersenzymes that remove histone or DNA modifications including histone and DNA demethylases, and (3) readersproteins that recognize a specific histone or DNA modification and modulate transcription by binding to these modifications. The bromodomain-containing family of proteins represents an important class of histone modification readers that recognize acetylated lysine residues and play critical roles in maintaining epigenetic memory and gene transcription (Dey et al. 2009; Zhao et al. 2011).

In this review, we briefly summarize the role of BET bromodomain proteins in various cancer types, the mechanisms of response and resistance to BET bromodomain inhibitors (BETis), and their clinical development.

\section{BET BROMODOMAIN PROTEINS}

The bromodomain was first described in 1992 as an evolutionarily conserved domain of $\sim 110$ amino acids present in several proteins in humans, fruit flies, and yeast with important roles in transcription (Haynes et al. 1992). Its structure consists of four $\alpha$ helices separated by variable loop regions, which together form a hydrophobic cavity that recognizes acetyllysine. The human genome encodes for 42 bromodomain-containing proteins with 61 unique bromodomains, where differences in the amino acid residues around the acetyllysine binding site impart binding specificity (Filippakopoulos and Knapp 2012). The BET (bromodomain and extraterminal domain) subfamily of bromodomain proteins, composed of BRD2, $\mathrm{BRD} 3, \mathrm{BRD} 4$, and BRDT, perform diverse roles in regulating transcription by RNA polymerase II (POLII) (Dhalluin et al. 1999). Common to all four BET proteins are two conserved amino-terminal bromodomains (BD1 and BD2) that are critical for their chromatin binding via recognition of acetylated lysine residues on histone tails and other nuclear proteins. A well-studied member of the BET family is BRD4, which was first identified as a MED1-interacting protein and a component of the mammalian Mediator complex (Jiang et al. 1998). Consistent with this interaction, BRD4 and Mediator co-occupy similar sites across the genome and can stabilize one another's occupancy at certain regions, including thousands of enhancers and promoters associated with active genes (Loven et al. 2013). Exceptionally high levels of these factors occur at a small set of large enhancer regions defined as super-enhancers (SEs). SEs drive genes that are essential for establishing and maintaining cellular identity and also play key roles in driving the expression of oncogenes during cancer development and progression (Chapuy et al. 2013; Hnisz et al. 2013; Loven et al. 2013). BRD4 is also a crucial regulator of transcriptional elongation that recruits the positive transcription elongation factor $\mathrm{b}(\mathrm{P}-\mathrm{TEFb})$ complex to chromatin and mediates the (C) 2016 Shu and Polyak. This article is distributed under the terms of the Creative Commons Attribution License, which permits unrestricted reuse and
redistribution provided that the original author and source are credited. 
formation of the active form of P-TEFb, which phosphorylates and activates RNA polymerase II. The BRD4/P$\mathrm{TEFb}$ interaction is important for rapid transcriptional reinitiation after mitosis (Jang et al. 2005; Yang et al. 2005).

A number of selective and potent small-molecule inhibitors have been developed that can compete for the acetyl-binding pockets present in the bromodomains of BET proteins and block gene expression (Filippakopoulos et al. 2010; Nicodeme et al. 2010). One of the most notable inhibitors is JQ1, a thienodiazepine-based small molecule that shows excellent inhibition against the BET subfamily in the low-nanomolar range and is especially effective against BRD4 (Filippakopoulos et al. 2010). The crystal structure of JQ1 and the first bromodomain of BRD4 showed excellent structural complementarity between the small molecule and the protein. JQ1 engages the bromodomain pocket in a manner that is competitive with acetylated peptide binding, thereby causing the displacement of BET proteins from chromatin with high affinity and specificity and excellent cell permeability. The development of these inhibitors has revealed novel insights into the physiological role and therapeutic potential of inhibiting BET function, especially in various human cancers and systemic inflammatory conditions (Nicodeme et al. 2010; Delmore et al. 2011; Zuber et al. 2011). Treatment of tumor cells with the BETi JQ1 causes a preferential loss of BRD4, Mediator, and $\mathrm{P}-\mathrm{TEFb}$ at SEs, leading to the relatively selective decrease of transcription at SE-associated genes, including the c-MYC oncogene in c-MYC-driven malignancies (Delmore et al. 2011; Loven et al. 2013). Thus, key oncogenic drivers can be regulated by SEs, which can confer disproportionate sensitivity to loss of BRD4 leading to rather selective inhibition of transcription by BETis. Further investigations have now shown that BETis can repress the expression of oncogenic transcription factors beyond c-MYC, thus expanding their potential clinical utility to several solid tumors, including colorectal cancer, castration-resistant prostate cancer, glioblastoma, non-small cell lung cancer, N-MYC-amplified neuroblastoma, ovarian carcinomas, and breast cancer (Lockwood et al. 2012; Puissant et al. 2013; Sahai et al. 2014, 2016; Shu et al. 2016). BRD4 was also identified as a component of a recurrent $\mathrm{t}(15 ; 19)$ chromosomal translocation in NUT midline carcinoma, an aggressive form of human squamous carcinoma (French et al. 2008). The resulting BRD4-NUT oncoprotein is an aberrant transcriptional regulator that relies on the bromodomains of BRD4 for its oncogenic function. JQ1 treatment induces differentiation in patient-derived NUT cancer cell lines and also showed excellent efficacy in xenograft models without obvious toxicity (Filippakopoulos et al. 2010).

To date, 10 BETis have entered clinical trials (Galdeano and Ciulli 2016). One of them, RVX-208 (Resverlogic) has reached Phase III clinical trials in a total of seven clinical trials for the treatment of atherosclerosis and associated cardiovascular disease (Galdeano and Ciulli 2016). Three additional BETis have also reached Phase II clinical trials (OTX015/MK-8628 [Merck],
BMS-9861158 [BMS], and GSK525762 [GSK]), while six other BETis have completed or are in ongoing Phase I clinical trials (TEN-010 [Roche], CPI-0610 [Constellation], GS-5829 [Gilead Sciences], BAY1238097 [Bayer], ABBV-075 [AbbVie], and INCB054329 [Incyte]), for both solid tumors and hematological malignancies. Clinical responses have been seen in NUT midline carcinoma patients and in leukemia, lymphoma, and multiple myeloma, primarily as a result of repressing c-MYC expression (Stathis et al. 2016). Additional trials are planned testing BETis in combination with chemotherapy, other targeted therapies, and immunotherapy.

\section{BET INHIBITORS IN BREAST CANCER}

Breast cancer is currently the second leading cause of cancer-related deaths in women worldwide. More than 200,000 women in the United States and more than one million across the world are diagnosed with breast cancer each year. Despite improvements in adjuvant therapy, many women still experience recurrences of their cancer sometimes even decades after initial diagnosis and treatment. Once metastatic, breast cancer is generally incurable and is responsible for approximately 40,000 deaths in the United States and more than 400,000 deaths worldwide annually (Siegel et al. 2016). Molecular interrogation of human breast cancers has led to the identification of at least four distinct tumor subtypes distinguished by their gene expression profiles and clinical behavior: luminal A, luminal B, HER2 ${ }^{+}$, and basal-like (Perou et al. 2000; Sorlie et al. 2001). These molecular subtypes complement, but do not fully overlap with pathologic classification based on the expression of estrogen receptor (ER), progesterone receptor (PR), and HER2. Luminal tumors are typically $\mathrm{ER}^{+} \mathrm{PR}^{+}$, and basal-like tumors are commonly "triple-negative", (ER ${ }^{-}, \mathrm{PR}^{-}$, and $\mathrm{HER} 2^{-}$), whereas HER2 ${ }^{+}$tumors have amplification of the ERBB2 oncogene.

$\mathrm{ER}^{+}$breast cancers represent $\sim 70 \%$ of all breast cancers and depend on estrogen-induced activation of ER for their growth. Estrogen (E2)-induced transcription requires coordinated recruitment of estrogen receptor $\alpha$ $(\mathrm{ER} \alpha)$ and multiple factors at the promoter of activated genes. However, the precise mechanism by which this complex stimulates the activity of RNA polymerase II required to execute transcription is largely unresolved. Genome-wide studies revealed an enrichment of BRD4 at transcriptional start sites of active genes and a requirement of BRD4 for H2B mono-ubiquitination in the transcribed regions of estrogen-responsive genes (Nagarajan et al. 2014). Importantly, BRD4 occupies distal EREs enriched for the histone H3 lysine 27acetyl (H3K27ac) mark and regulates enhancer RNA synthesis by affecting RNAPII recruitment and elongation. Consistently, BRD4 activity is required for the proliferation of $\mathrm{ER}^{+}$breast and endometrial cancer cells and for uterine growth in mice (Nagarajan et al. 2014). JQ1 suppresses E2-induced growth and transcription in MCF7 and T47D ER ${ }^{+}$breast cancer cell lines (Nagarajan et al. 2014). JQ1 treatment 
does not disrupt E2-induced recruitment of ER, SRC3, or histone acetylation status at the promoters of genes but blocks the E2-induced transition of RNA polymerase II from initiation to elongation by stalling it at the promoter region of the responsive genes upstream of the transcription start site.

Treatment options for $\mathrm{ER}^{+}$breast cancer patients have relied heavily on antihormonal strategies with varying anti-estrogen modalities including SERMs (selective estrogen receptor modulators), such as tamoxifen and raloxifen, and aromatase inhibitors (Early Breast Cancer Trialists' Collaborative Group 2005). Although most patients respond to endocrine agents, a significant fraction will eventually relapse (Lupien et al. 2010). A number of studies have investigated the potential use of BETis in endocrine-resistant breast tumors. BET bromodomain proteins contribute to tamoxifen resistance by recruiting WHSC1, a histone H3K6 methyltransferase, to ER $\alpha$ for facilitating $E R \alpha$ gene expression (Feng et al. 2014). JQ1 potently suppresses the classic ER $\alpha$ signaling pathway and the growth of tamoxifen-resistant breast cancer cells in vitro and in vivo (Feng et al. 2014). Importantly, tamoxifen-resistant cell lines are more sensitive to the BETi JQ1 than are parental lines, implying its potential use as a combination agent to overcome endocrine resistance.

The PI3K/mTOR pathway has also been shown to mediate resistance to anti-estrogens in $\mathrm{ER}^{+}$breast cancer patients (Miller et al. 2010). Everolimus, an allosteric inhibitor of mTOR complex 1, has been approved in combination with exemestane (an aromatase inhibitor) for the treatment of breast cancer patients with ER ${ }^{+}$HER2 ${ }^{-}$ tumors who progressed on anastrozole or letrozole aromatase inhibitor treatment alone (Baselga et al. 2012). However, despite initial efficacy, breast tumors develop acquired resistance to everolimus. Acquired resistance to $\mathrm{PI} 3 \mathrm{~K} / \mathrm{mTOR} /$ Akt pathway inhibitors is often associated with compensatory feedback loops involving the activation of oncogenes (Piccart et al. 2014). c-MYC was identified as a driver of mTOR inhibitor resistance in longterm estrogen deprivation models (Bihani et al. 2015). Both everolimus resistance and acute treatment of everolimus can lead to the up-regulation of MYC expression and the enrichment of MYC signatures. Importantly, depletion of c-MYC using siRNA or the BET inhibitor JQ1 restored everolimus sensitivity, whereas a combination of everolimus and JQ1 led to synergistic growth inhibition in 3D Matrigel cultures and in xenograft models.

BETis were also shown to overcome resistance to class I PI3K inhibitors in a murine model of metastatic breast cancer driven by mutant PI3K and MYC (Stratikopoulos et al. 2015). BETis decreased PI3K signaling and dissociated BRD4 from chromatin at genomic regions that regulate the expression of insulin receptor and epidermal growth factor receptor (EGFR) family receptor tyrosine kinases (RTKs). Combined PI3K and BET inhibition induced cell death, tumor regression, and clamped inhibition of PI3K signaling.

The $E R B B 2$ oncogene is amplified or overexpressed in $\sim 25 \%$ of breast cancers and serves as the primary driver of HER2 ${ }^{+}$tumors. Therapeutics that target HER2, such as trastuzumab (Herceptin) and lapatinib, often provide initial clinical benefit, but resistance frequently develops. Resistance to lapatinib can result from kinome signaling reprograming in tumor cells including up-regulation of HER 3 and activation of multiple tyrosine kinases. Genetic and chemical inhibition of BET bromodomain proteins suppresses transcription of many lapatinib-induced kinases involved in resistance, including ERBB3, IGF1R, DDR1, MET, and fibroblast growth factor receptors (FGFRs), preventing downstream SRC/FAK signaling and AKT reactivation (Stuhlmiller et al. 2015). In line with this, combination treatment with lapatinib and JQ1 blocked the growth of lapatinib-resistant cells. Thus, BETis may also be useful in decreasing resistance to $\mathrm{HER}^{+}{ }^{+}$targeted therapies in HER2 ${ }^{+}$breast cancer.

\section{BET INHIBITORS IN TRIPLE-NEGATIVE BREAST CANCER}

Triple-negative breast cancers (TNBCs) that are negative for ER, PR, and HER2 account for 20\% of breast cancers in the United States. TNBCs have a high rate of recurrence to distant metastatic sites and are currently the only major breast tumor subtype that lacks targeted therapies (Lehmann et al. 2011). Hence, new therapeutic options are clearly needed. We hypothesized that because of the central role for c-MYC in TNBCs (Daemen et al. 2013), these tumors may be dependent on BET bromodomain proteins. To test this hypothesis, we screened a large panel of breast cancer cell lines with BETis and found that TNBC cell lines showed significant growth inhibition in cell culture when treated with low-nanomolar concentrations of BETis, compared with luminal cell lines, the majority of which were relatively resistant (Shu et al. 2016). The growth of established xenografts derived from TNBC cell lines and from primary patient TNBC samples was also efficiently inhibited by JQ1 treatment. The expression of BRD4 is higher in TNBCs compared with luminal lines, and its down-regulation by TET-inducible short-hairpin RNAs (shRNAs) arrested TNBC cell growth in vitro whereas in vivo it led to complete tumor regression without any regrowth even after stopping doxycycline treatment. c-MYC is more abundantly expressed in TNBC lines compared with luminal ones, but its expression did not correlate with JQ1 response and did not change consistently after JQ1 treatment. In line with the role of BRD4 in transcription restart after $G_{2} / M$, JQ1 treatment prevented cell cycle reentry, arrested TNBC cells in early $\mathrm{G}_{1}$, and induced apoptosis, senescence, and luminal features in basal-like breast cancer cell lines. Using integrated epigenomic analysis, JQ1 was found to efficiently displace chromatin-bound BRD4 in sensitive TNBC cells. This effect was more pronounced at genomic regions showing elevated Bio-JQ1 binding based on Chem-seq, implying strong association between BRD4 and JQ1 genomic binding patterns. Genome-wide studies with ChIP-seq and RNA-seq have also revealed preferential disruption of SE-associated genes by JQ1, leading to deregulation of coordinated transcriptional pathways involved in cell proliferation, 


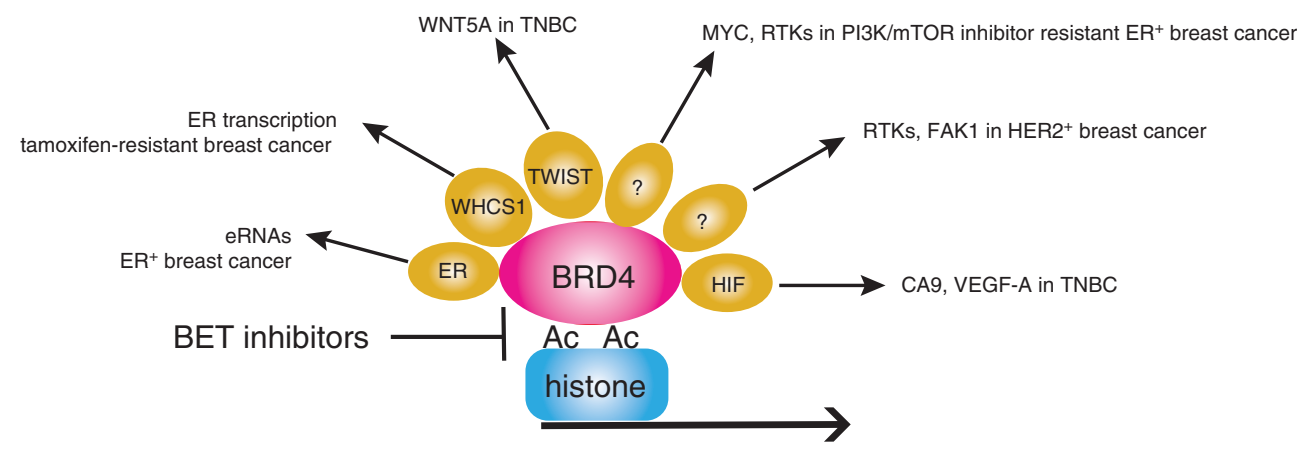

Figure 1. Schematic model of BRD4 mechanism of action in breast cancer. BRD4 binds to the indicated proteins leading to activation of downstream genes. ER, estrogen receptor; TNBC, triple-negative breast cancer; RTKs, receptor tyrosine kinases; HER2, human epidermal growth factor receptor 2; VEGF-A, vascular endothelial growth factor A; HIF, hypoxia-inducible factor.

invasion, and survival. These results provide strong preclinical data indicating that BETis may represent novel therapeutic agents for the treatment of TNBCs.

Hypoxia enables progression of TNBCs partly by driving metabolic adaptation, angiogenesis, and metastasis through up-regulation of hypoxia-regulated genes (da Motta et al. 2017). JQ1 treatment significantly down-regulates the hypoxic transcriptome response including CA9 and VEGF-A by preventing HIF binding to the hypoxia response element in CA9 promoter but does not alter HIF expression or activity (da Motta et al. 2017). JQ1 reduced TNBC growth in vitro and in vivo and inhibited xenograft vascularization. Zhou and his colleagues showed that the binding of BRD4 with diacetylated TWIST directs WNT5A expression in basal-like breast cancer (BLBC) (Shi et al. 2014). TIP60 diacetylates TWIST at its "histone H4-mimic" GK-X-GK motif, which binds to the second bromodomain of BRD4, whose first bromodomain interacts with acetylated $\mathrm{H} 4$, thereby constructing an activated TWIST/BRD4/P-TEFb/RNA-Pol II complex at the WNT5A promoter and enhancer. JQ1 treatment disrupted the TWIST-BRD4 interaction and resulted in significant WNT5A reduction, leading to inhibition of invasion, cancer stem cell (CSC)-like properties and tumorigenicity of BLBC. TWIST-BRD4 interaction is required for the recruitment of BRD4 at the WNT5A SE to activate WNT5A transcription. Histone H4 and TWIST synergistically interact with BRD4. Overall, they concluded that the TWIST-BRD4-WNT5A axis is critical for the growth of BLBC in vitro and in vivo.

Based on these studies BETis appear to be promising novel therapeutic agents in all types of breast tumors, especially in combination with other therapies, with underlying mechanisms varying depending on tumor subtype (Fig. 1).

\section{MECHANISMS OF BETi RESISTANCE}

Targeted therapies inevitably select for resistance during prolonged treatment. Furthermore, studying mechanisms of acquired resistance can also help delineate the detailed mechanism of action of a particular agent. Therefore, we and others have investigated potential resistance mechanisms to BETis in various cancer types and iden- tified various mechanisms of resistance in different cancer types (Fig. 2). In TNBC, we identified a unique mechanism of epigenomic resistance to BETi in paired cell lines selected for acquired resistance to BET inhibition from previously sensitive TNBCs by long-term culture in escalating doses of JQ1 (Shu et al. 2016). BETi resistance was not attributable to drug export or alterations of BET bromodomain proteins and other driver genes, and resistant cells maintained their dependency on BRD4. By comparing Bio-JQ1 Chem-seq profiles of sensitive and resistant cells, we found a significant gain in the number of SEs in resistant cells, and a less pronounced loss of fewer SEs. The gain of Bio-JQ1 SEs was associated with gain of BRD4 binding to these genomic loci and also with increased transcription of the associated genes. Among the top gained super-enhancers in JQ1-resistant SUM159 cells was an upstream and intragenic region of $\mathrm{H} 3 \mathrm{~K} 27 \mathrm{ac}$ enrichment at the $B C L-x L$ locus, consistent with higher BCL-XL expression in resistant cells. BRD4 is not displaced from chromatin following JQ1 treatment in resistant cells because of the recruitment of BRD4 to SE loci in a bromodomain-independent manner. By performing an unbiased proteomic analysis using RIME (rapid immunoprecipitation mass spectrometry of endogenous protein) (Mohammed et al. 2013), we identified strong association of BRD4 with MED1 in resistant cells. Cheng-Ming Chiang's group has previously reported that BRD4 is phosphorylated at several residues by casein kinase II (CK2) and this increases the stability and nuclear localization of BRD4 (Wu et al. 2013). We found a marked increase of phospho-BRD4 (pBRD4) in resistant cells and also identified PP2A as a BRD4 phosphatase. We determined that BRD4 is hyperphosphorylated in JQ1-resistant cells and this increases the recruitment of BRD4 to MED1 and contributes to BETi resistance. We confirmed this by expressing BRD4 mutants that cannot be phosphorylated (7A) or that mimic constitutive phosphorylation (7D) and showing that these increase and decrease BETi sensitivity, respectively. Last, based on our genomic and proteomic profiling of resistant cells, we identified potential drug combinations that can overcome BETi resistance. Specifically, inhibitors of BCL-XL (e.g., ABT263) and CK2 (e.g., CX-4945) and activators of PP2A (e.g., perphena- 
Sensitive cells

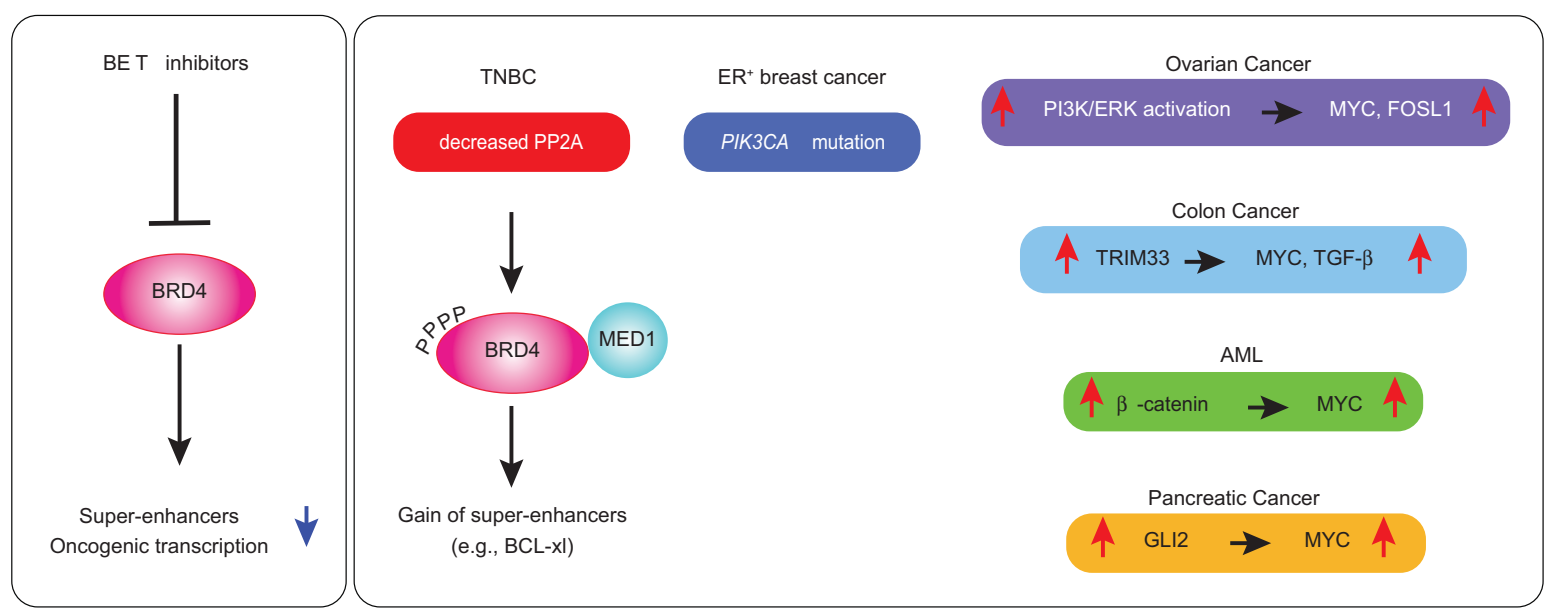

Figure 2. BET bromodomain inhibitor (BETi) resistance mechanisms. In sensitive cells BETis inhibit BRD4 function through disrupting its binding to super-enhancers, thereby turning off downstream oncogenic transcription. The model depicts mechanisms of BETi resistance in the indicated cancer types. TNBC, triple-negative breast cancer; ER, estrogen receptor; PI3K, phosphoinositide 3-kinase; TRIM33, tripartite motif-containing protein 33.

zine) synergize with JQ1 and can overcome BETi resistance in TNBCs.

Ben Neel's group has also identified BRD4 as a potential target in luminal breast cancers by performing an shRNA screen and integrated molecular analyses in a large panel of breast cancer cell lines (Marcotte et al. 2016). They tested the effect of JQ1 in a subset of the cell lines used in the shRNA screen and found that lines with high JQ1 sensitivity underwent apoptosis, whereas resistant lines had slower cell cycle progression. Many luminal HER2 ${ }^{+}$breast cancer cell lines sensitive to BRD4 down-regulation were JQ1-resistant, but most basal-like lines that were sensitive to BRD4 loss were JQ1-sensitive. JQ1 sensitivity did not reflect impaired MYC expression, and exogenous MYC did not convert JQ1-sensitive lines to JQ1-resistant ones. Integrative analysis revealed a strong correlation between JQ1 resistance and PIK3CA mutation. Overexpression of wild-type or mutant $P I K 3 C A$ conferred JQ1 resistance in JQ1-sensitive cells. PI3Kspecific kinase inhibitors increased the JQ1 sensitivity of resistant cells in vitro and in vivo.

In pancreatic cancer cells, acquired resistance to BETis was found to be associated with resistance to BRD4 down-regulation, increased expression of JQ1 targets including c-MYC, FOSL1, and HMGA2, and epithelialmesenchymal transition (EMT) (Kumar et al. 2015). JQ1 resistant cells remain dependent on c-MYC through increased binding of GLI2 to the $c-M Y C$ promoter. Targeting GLI2 resensitized pancreatic cancer cells to BET inhibitors and BRD4 down-regulation.

To identify potential mediators of BETi resistance in MLL-AF9-driven AML (acute myeloid leukemia), Johannes Zuber's group performed a chromatin-focused RNAi screen in a BETi-sensitive MLL-AF9;Nras ${ }^{\text {G12D }}$ driven AML mouse cell line (Rathert et al. 2015). They found that resistance to BET bromodomain inhibitors is associated with profound changes in the enhancer landscape. The suppression of the PRC2 complex promotes
BET inhibitor resistance in AML by facilitating the remodeling of regulatory pathways that restore the transcription of key targets such as MYC. This process involves the activation of a focal MYC enhancer that activates WNT genes in response to BET inhibition. WNT signaling promotes primary and acquired BETi resistance in leukemia, establishing it as a major driver and candidate biomarker of BETi resistance in leukemia.

Mark A. Dawson's group derived BETi-resistant mouse AML cells by continuously exposing the cells to BET inhibitor eventually yielding drug-resistant clonal populations in vitro and in vivo (Fong et al. 2015). BETi resistance in this model is not mediated by increased drug efflux or metabolism but was shown to emerge from leukemia stem cells, suggesting that the AML cancer-stem-cell population, or a subset thereof, does not respond to BET inhibitors. Chromatin-bound BRD4 is globally reduced in resistant cells, whereas the expression of key target genes such as Myc remains unaltered. They also showed that $\mathrm{Wnt} / \beta$-catenin activation has a role in BETi resistance and inhibition of $\mathrm{WNT} / \beta$-catenin signaling restores BETi sensitivity in vitro and in vivo.

In colon cancer, loss of tripartite motif-containing protein 33 (TRIM33), a chromatin-associated E3 ubiquitin ligase, was identified to confer resistance to BETi based on shRNA-based genetic screen (Shi et al. 2016). TRIM33 silencing reprograms cancer cells to a more resistant state through at least two mechanisms. TRIM33 loss maintains MYC expression following BETi treatment. Moreover, TRIM33 silencing enhances TGF- $\beta$ receptor expression and signaling. The authors also showed that inhibition of TGF- $\beta$ signaling increased sensitivity to BET bromodomain inhibitors.

The James S. Duncan group reported that BETi resistance in ovarian cancers is associated with adaptive kinome reprogramming (Kurimchak et al. 2016). Despite initial inhibition by JQ1, ovarian carcinoma (OC) cells acquired resistance following sustained treatment with BETi. Using 
a mass spectrometry approach that globally measures kinase signaling at the proteomic level, they investigated changes following JQ1 treatment in a panel of BRD4-dependent OC cell lines. Ovarian cancer cells chronically exposed to JQ1 acquire dependency on RTK, PI3K, and/ or ERK signaling and show exquisite sensitivity to kinase inhibitors targeting these pathways. Elevated PI3K/ERK activity stabilizes MYC/FOSL1 proteins in JQ1-resistant cells. Cotargeting BET proteins and RTK, PI3K or ERK signaling enhances BET inhibitor therapy.

In summary, it appears that resistance to BETis might be due to nongenetic mechanisms and involves the activation of different pathways or downstream genes depending on tumor type.

\section{CONCLUSION}

BET bromodomain inhibitors are being explored as promising therapeutic agents in numerous hematopoietic and solid tumor types including NUT midline carcinoma, leukemia, and breast cancer with several ongoing clinical trials actively enrolling patients. BET inhibitors may also have a role in preventing or overcoming acquired resistance to U.S. Federal Drug Administration (FDA)-approved targeted therapies such as endocrine therapy and $\mathrm{PI} 3 \mathrm{~K} / \mathrm{mTOR}$ inhibitors in $\mathrm{ER}^{+}$breast cancer patients, and HER2-targeted therapy in HER2 ${ }^{+}$breast cancers. Furthermore, as mechanisms of BETi resistance have been investigated in several cancer types, combination treatment with inhibitors of pathways mediating resistance may significantly improve the clinical efficacy of BETis. Finally, several studies have shown that BRD4 regulates the expression of PD-L1 and CD47 immune regulatory proteins in ovarian cancer, leukemia, and lymphomas, and BETi treatment increases cytotoxic T-cell activity (Casey et al. 2016; Kagoya et al. 2016; Zhu et al. 2016). Therefore, the antitumor effects of BET inhibitors may include the targeting of both tumor cells as well as the tumor-promoting immune environment. Thus, combination therapy of BETi with immune checkpoint blockade may provide new opportunities to increase the efficacy of immunotherapies.

\section{ACKNOWLEDGMENTS}

We thank members of our laboratory for their critical reading of our manuscript and helpful discussions. S.S. is funded by a Susan G. Komen Postdoctoral Fellowship. This work was supported by the National Institutes of Health (P50CA168504) SPORE in Breast Cancer and the Susan G. Komen Foundation.

Conflict of interest: The authors have no conflicts of interest to declare.

\section{REFERENCES}

Baselga J, Campone M, Piccart M, Burris HA III, Rugo HS, Sahmoud T, Noguchi S, Gnant M, Pritchard KI, Lebrun F, et al. 2012. Everolimus in postmenopausal hormone-receptorpositive advanced breast cancer. NEngl J Med 366: 520-529.
Bihani T, Ezell SA, Ladd B, Grosskurth SE, Mazzola AM, Pietras M, Reimer C, Zinda M, Fawell S, D'Cruz CM. 2015. Resistance to everolimus driven by epigenetic regulation of MYC in $\mathrm{ER}^{+}$breast cancers. Oncotarget 6: 2407-2420.

Casey SC, Tong L, Li Y, Do R, Walz S, Fitzgerald KN, Gouw AM, Baylot V, Gutgemann I, Eilers M, et al. 2016. MYC regulates the antitumor immune response through CD47 and PD-L1. Science 352: 227-231.

Chapuy B, McKeown MR, Lin CY, Monti S, Roemer MG, Qi J, Rahl PB, Sun HH, Yeda KT, Doench JG, et al. 2013. Discovery and characterization of super-enhancer-associated dependencies in diffuse large B cell lymphoma. Cancer Cell 24: 777-790.

Daemen A, Griffith OL, Heiser LM, Wang NJ, Enache OM, Sanborn Z, Pepin F, Durinck S, Korkola JE, Griffith M, et al. 2013. Modeling precision treatment of breast cancer. Genome Biol 14: R110.

Dalgliesh GL, Furge K, Greenman C, Chen L, Bignell G, Butler A, Davies H, Edkins S, Hardy C, Latimer C, et al. 2010. Systematic sequencing of renal carcinoma reveals inactivation of histone modifying genes. Nature 463: 360-363.

da Motta LL, Ledaki I, Purshouse K, Haider S, De Bastiani MA, Baban D, Morotti M, Steers G, Wigfield S, Bridges E, et al. 2017. The BET inhibitor JQ1 selectively impairs tumour response to hypoxia and downregulates CA9 and angiogenesis in triple negative breast cancer. Oncogene 36: 122-132.

Delmore JE, Issa GC, Lemieux ME, Rahl PB, Shi J, Jacobs HM, Kastritis E, Gilpatrick T, Paranal RM, Qi J, et al. 2011. BET bromodomain inhibition as a therapeutic strategy to target c-Myc. Cell 146: 904-917.

Dey A, Nishiyama A, Karpova T, McNally J, Ozato K. 2009. Brd4 marks select genes on mitotic chromatin and directs postmitotic transcription. Mol Biol Cell 20: 4899-4909.

Dhalluin C, Carlson JE, Zeng L, He C, Aggarwal AK, Zhou MM. 1999. Structure and ligand of a histone acetyltransferase bromodomain. Nature 399: 491-496.

Early Breast Cancer Trialists' Collaborative Group. 2005. Effects of chemotherapy and hormonal therapy for early breast cancer on recurrence and 15-year survival: An overview of the randomised trials. Lancet 365: $1687-1717$.

Feng Q, Zhang Z, Shea MJ, Creighton CJ, Coarfa C, Hilsenbeck SG, Lanz R, He B, Wang L, Fu X, et al. 2014. An epigenomic approach to therapy for tamoxifen-resistant breast cancer. Cell Res 24: 809-819.

Filippakopoulos P, Knapp S. 2012. The bromodomain interaction module. FEBS Lett 586: 2692-2704.

Filippakopoulos P, Qi J, Picaud S, Shen Y, Smith WB, Fedorov O, Morse EM, Keates T, Hickman TT, Felletar I, et al. 2010. Selective inhibition of BET bromodomains. Nature 468: 1067-1073.

Fong CY, Gilan O, Lam EY, Rubin AF, Ftouni S, Tyler D, Stanley K, Sinha D, Yeh P, Morison J, et al. 2015. BET inhibitor resistance emerges from leukaemia stem cells. Nature 525: 538-542.

French CA, Ramirez CL, Kolmakova J, Hickman TT, Cameron MJ, Thyne ME, Kutok JL, Toretsky JA, Tadavarthy AK, Kees UR, et al. 2008. BRD-NUT oncoproteins: A family of closely related nuclear proteins that block epithelial differentiation and maintain the growth of carcinoma cells. Oncogene 27: 2237-2242.

Galdeano C, Ciulli A. 2016. Selectivity on-target of bromodomain chemical probes by structure-guided medicinal chemistry and chemical biology. Future Med Chem 8: 1655-1680.

Haynes SR, Dollard C, Winston F, Beck S, Trowsdale J, Dawid IB. 1992. The bromodomain: A conserved sequence found in human, Drosophila and yeast proteins. Nucleic Acids Res 20: 2603.

Hnisz D, Abraham BJ, Lee TI, Lau A, Saint-Andre V, Sigova AA, Hoke HA, Young RA. 2013. Super-enhancers in the control of cell identity and disease. Cell 155: 934-947.

Jang MK, Mochizuki K, Zhou M, Jeong HS, Brady JN, Ozato K. 2005. The bromodomain protein Brd4 is a positive regulatory component of P-TEFb and stimulates RNA polymerase IIdependent transcription. Mol Cell 19: 523-534. 
Jiang YW, Veschambre P, Erdjument-Bromage H, Tempst P, Conaway JW, Conaway RC, Kornberg RD. 1998. Mammalian mediator of transcriptional regulation and its possible role as an end-point of signal transduction pathways. Proc Natl Acad Sci 95: 8538-8543.

Kagoya Y, Nakatsugawa M, Yamashita Y, Ochi T, Guo T, Anczurowski M, Saso K, Butler MO, Arrowsmith CH, Hirano N. 2016. BET bromodomain inhibition enhances T cell persistence and function in adoptive immunotherapy models. $J$ Clin Invest 126: 3479-3494.

Kumar K, Raza SS, Knab LM, Chow CR, Kwok B, Bentrem DJ, Popovic R, Ebine K, Licht JD, Munshi HG. 2015. GLI2dependent c-MYC upregulation mediates resistance of pancreatic cancer cells to the BET bromodomain inhibitor JQ1. Sci Rep 5: 9489.

Kurimchak AM, Shelton C, Duncan KE, Johnson KJ, Brown J, O’Brien S, Gabbasov R, Fink LS, Li Y, Lounsbury N, et al. 2016. Resistance to BET bromodomain inhibitors is mediated by kinome reprogramming in ovarian cancer. Cell Rep 16: $1273-1286$.

Lehmann BD, Bauer JA, Chen X, Sanders ME, Chakravarthy AB, Shyr Y, Pietenpol JA. 2011. Identification of human triplenegative breast cancer subtypes and preclinical models for selection of targeted therapies. J Clin Invest 121: 2750-2767.

Lockwood WW, Zejnullahu K, Bradner JE, Varmus H. 2012. Sensitivity of human lung adenocarcinoma cell lines to targeted inhibition of BET epigenetic signaling proteins Proc Natl Acad Sci 109: 19408-19413.

Loven J, Hoke HA, Lin CY, Lau A, Orlando DA, Vakoc CR, Bradner JE, Lee TI, Young RA. 2013. Selective inhibition of tumor oncogenes by disruption of super-enhancers. Cell 153: 320-334.

Lupien M, Meyer CA, Bailey ST, Eeckhoute J, Cook J, Westerling T, Zhang X, Carroll JS, Rhodes DR, Liu XS, et al. 2010. Growth factor stimulation induces a distinct $\mathrm{ER} \alpha$ cistrome underlying breast cancer endocrine resistance. Genes Dev 24: 2219-2227.

Marcotte R, Sayad A, Brown KR, Sanchez-Garcia F, Reimand J, Haider M, Virtanen C, Bradner JE, Bader GD, Mills GB, et al. 2016. Functional genomic landscape of human breast cancer drivers, vulnerabilities, and resistance. Cell 164: 293-309.

Miller TW, Hennessy BT, Gonzalez-Angulo AM, Fox EM, Mills GB, Chen H, Higham C, Garcia-Echeverria C, Shyr Y, Arteaga CL. 2010. Hyperactivation of phosphatidylinositol-3 kinase promotes escape from hormone dependence in estrogen receptor-positive human breast cancer. J Clin Invest 120: $2406-2413$.

Mohammed H, D'Santos C, Serandour AA, Ali HR, Brown GD, Atkins A, Rueda OM, Holmes KA, Theodorou V, Robinson $\mathrm{JL}$, et al. 2013. Endogenous purification reveals GREB1 as a key estrogen receptor regulatory factor. Cell Rep 3: 342-349.

Morin RD, Mendez-Lago M, Mungall AJ, Goya R, Mungall KL, Corbett RD, Johnson NA, Severson TM, Chiu R, Field M, et al. 2011. Frequent mutation of histone-modifying genes in non-Hodgkin lymphoma. Nature 476: 298-303.

Nagarajan S, Hossan T, Alawi M, Najafova Z, Indenbirken D, Bedi U, Taipaleenmaki H, Ben-Batalla I, Scheller M, Loges $\mathrm{S}$, et al. 2014. Bromodomain protein BRD4 is required for estrogen receptor-dependent enhancer activation and gene transcription. Cell Rep 8: 460-469.

Nicodeme E, Jeffrey KL, Schaefer U, Beinke S, Dewell S, Chung CW, Chandwani R, Marazzi I, Wilson P, Coste H, et al. 2010. Suppression of inflammation by a synthetic histone mimic. Nature 468: 1119-1123.

Perou CM, Sorlie T, Eisen MB, van de Rijn M, Jeffrey SS, Rees CA, Pollack JR, Ross DT, Johnsen H, Akslen LA, et al. 2000. Molecular portraits of human breast tumours. Nature 406: $747-752$.

Piccart M, Hortobagyi GN, Campone M, Pritchard KI, Lebrun F, Ito Y, Noguchi S, Perez A, Rugo HS, Deleu I, et al. 2014. Everolimus plus exemestane for hormone-receptor-positive, human epidermal growth factor receptor-2-negative advanced breast cancer: Overall survival results from BOLERO-2. Ann Oncol 25: 2357-2362.
Puissant A, Frumm SM, Alexe G, Bassil CF, Qi J, Chanthery YH, Nekritz EA, Zeid R, Gustafson WC, Greninger P, et al. 2013. Targeting MYCN in neuroblastoma by BET bromodomain inhibition. Cancer Discov 3: 308-323.

Rathert P, Roth M, Neumann T, Muerdter F, Roe JS, Muhar M, Deswal S, Cerny-Reiterer S, Peter B, Jude J, et al. 2015. Transcriptional plasticity promotes primary and acquired resistance to BET inhibition. Nature 525: 543-547.

Sahai V, Kumar K, Knab LM, Chow CR, Raza SS, Bentrem DJ, Ebine K, Munshi HG. 2014. BET bromodomain inhibitors block growth of pancreatic cancer cells in three-dimensional collagen. Mol Cancer Ther 13: 1907-1917.

Sahai V, Redig AJ, Collier KA, Eckerdt FD, Munshi HG. 2016. Targeting bet bromodomain proteins in solid tumors. Oncotarget doi: 10.18632/oncotarget.9804.

Shi J, Wang Y, Zeng L, Wu Y, Deng J, Zhang Q, Lin Y, Li J, Kang T, Tao M, et al. 2014. Disrupting the interaction of BRD4 with diacetylated Twist suppresses tumorigenesis in basal-like breast cancer. Cancer Cell 25: 210-225.

Shi X, Mihaylova VT, Kuruvilla L, Chen F, Viviano S, Baldassarre M, Sperandio D, Martinez R, Yue P, Bates JG, et al. 2016. Loss of TRIM33 causes resistance to BET bromodomain inhibitors through MYC- and TGF- $\beta$-dependent mechanisms. Proc Natl Acad Sci 113: E4558-E4566.

Shu S, Lin CY, He HH, Witwicki RM, Tabassum DP, Roberts JM, Janiszewska M, Huh SJ, Liang Y, Ryan J, et al. 2016. Response and resistance to BET bromodomain inhibitors in triple-negative breast cancer. Nature 529: 413-417.

Siegel RL, Miller KD, Jemal A. 2016. Cancer statistics, 2016. CA Cancer J Clin 66: 7-30.

Sorlie T, Perou CM, Tibshirani R, Aas T, Geisler S, Johnsen H, Hastie T, Eisen MB, van de Rijn M, Jeffrey SS, et al. 2001. Gene expression patterns of breast carcinomas distinguish tumor subclasses with clinical implications. Proc Natl Acad Sci 98: 10869-10874.

Stathis A, Zucca E, Bekradda M, Gomez-Roca C, Delord JP, de La Motte Rouge T, Uro-Coste E, de Braud F, Pelosi G, French CA. 2016. Clinical response of carcinomas harboring the BRD4-NUT oncoprotein to the targeted bromodomain inhibitor OTX015/MK-8628. Cancer Discov 6: 492-500.

Stratikopoulos EE, Dendy M, Szabolcs M, Khaykin AJ, Lefebvre C, Zhou MM, Parsons R. 2015. Kinase and BET inhibitors together clamp inhibition of PI3 K signaling and overcome resistance to therapy. Cancer Cell 27: 837-851.

Stuhlmiller TJ, Miller SM, Zawistowski JS, Nakamura K, Beltran AS, Duncan JS, Angus SP, Collins KA, Granger DA, Reuther RA, et al. 2015. Inhibition of Lapatinib-induced kinome reprogramming in ERBB2-positive breast cancer by targeting BET family bromodomains. Cell Rep 11: 390-404.

van Haaften G, Dalgliesh GL, Davies H, Chen L, Bignell G, Greenman C, Edkins S, Hardy C, O'Meara S, Teague J, et al. 2009. Somatic mutations of the histone H3K27 demethylase gene UTX in human cancer. Nat Genet 41: 521-523.

Wu SY, Lee AY, Lai HT, Zhang H, Chiang CM. 2013. Phospho switch triggers Brd4 chromatin binding and activator recruitment for gene-specific targeting. Mol Cell 49: 843-857.

Yang Z, Yik JH, Chen R, He N, Jang MK, Ozato K, Zhou Q. 2005. Recruitment of P-TEFb for stimulation of transcriptional elongation by the bromodomain protein Brd4. Mol Cell 19: 535-545.

Zhao R, Nakamura T, Fu Y, Lazar Z, Spector DL. 2011. Gene bookmarking accelerates the kinetics of post-mitotic transcriptional re-activation. Nat Cell Biol 13: 1295-1304.

Zhu H, Bengsch F, Svoronos N, Rutkowski MR, Bitler BG, Allegrezza MJ, Yokoyama Y, Kossenkov AV, Bradner JE, Conejo-Garcia JR, et al. 2016. BET bromodomain inhibition promotes anti-tumor immunity by suppressing PD-L1 expression. Cell Rep 16: 2829-2837.

Zuber J, Shi J, Wang E, Rappaport AR, Herrmann H, Sison EA, Magoon D, Qi J, Blatt K, Wunderlich M, et al. 2011. RNAi screen identifies Brd4 as a therapeutic target in acute myeloid leukaemia. Nature 478: 524-528. 


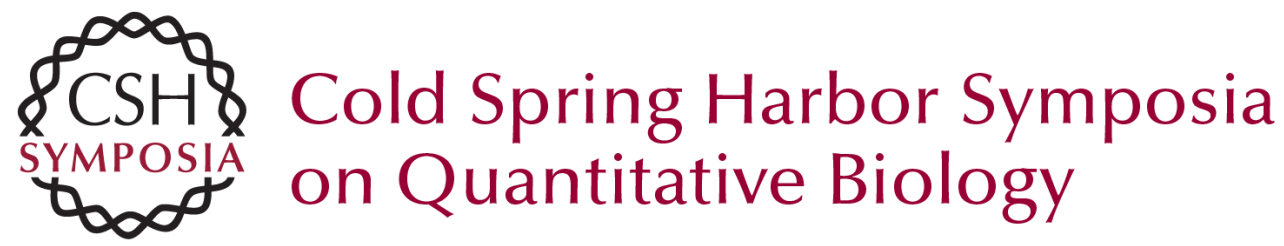

\section{BET Bromodomain Proteins as Cancer Therapeutic Targets}

Shaokun Shu and Kornelia Polyak

Cold Spring Harb Symp Quant Biol 2016 81: 123-129 originally published online January 6, 2017 Access the most recent version at doi:10.1101/sqb.2016.81.030908

References This article cites 54 articles, 10 of which can be accessed free at: http://symposium.cshlp.org/content/81/123.full.html\#ref-list-1
Creative This article is distributed under the terms of the
Commons http://creativecommons.org/licenses/by/4.0/, which permits unrestricted
License reuse and redistribution provided that the original author and source are credited.

Email Alerting Receive free email alerts when new articles cite this article - sign up in Service the box at the top right corner of the article or click here. 